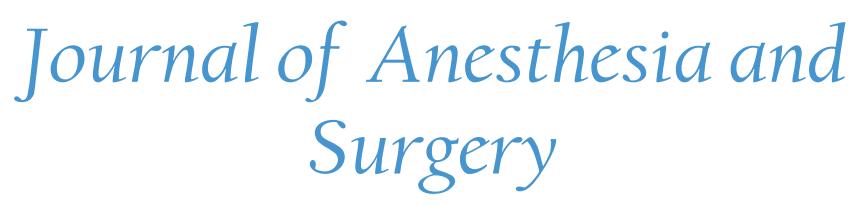

\title{
Functional Outcomes and Health Related Quality of Life after Artificial Urinary Sphincter Implantation: Evaluation with Validated Questionnaires
}

\author{
M.A. Cerruto ${ }^{1}$, C. D'Elia ${ }^{2 *}$, A. Minja ${ }^{1}$, A.B. Porcaro ${ }^{1}$, M. Balzarro ${ }^{1}$, A. Sarti ${ }^{1}$, E. Rubilotta ${ }^{1}$, S. Siracusa- \\ no ${ }^{1}$, W. Artibani ${ }^{1}$
}

${ }^{1}$ Urology Department, Azienda Ospedaliera Universitaria di Verona, Verona, Italy

${ }^{2}$ Urology Department, Bolzano General Hospital, Bolzano, Italy

"Corresponding author: Carolina D'Elia, Urology Department, Bolzano General Hospital, 39100 Bolzano, Italy

Tel: +39-349-6707889; E-mail: karolinedelia@gmail.com

\begin{abstract}
Introduction: Urinary incontinence ranges from 4 to $31 \%$ after prostatic surgery. The aim of our study was to assess efficacy and quality of life outcomes of 37 patients who underwent artificial urinary sphincter AMS (American Medical System) 800 placement in a single academic urologic clinic.

Materials and methods: Pre and post operative stress urinary incontinence (SUI) was evaluated using the daily pad use (pad per day used PPD) and the Italian validated International Consultation on Incontinence Questionnaire - short form (ICIQ-SF), whereas health related quality of life (HRQoL) and subjective satisfaction of the patients was evaluated with the Italian validated Patient Global Impression of Improvement (PGI-I) questionnaire.

Results: Median preoperative PPD used was 4; after a median follow up of 4 years, median PPD used was 1 . With regard to ICIQ-SF questionnaire, 4 patients $(12.5 \%)$ responded that they never lose urine and $22(68.76 \%)$ only during exercise and / or sneezing. Moreover, median PGI-1 score was 1, documenting a better HRLQoL after AMS positioning. With regard to the answer regarding improvement after surgery, median score was 90 , while median score concerning satisfaction was 99 . When we asked, "Would you recommend the post to a friend?" only 1 patient replied no.

Conclusions: AMS 800 ensures good results in terms of urinary continence and a satisfactory quality of life.
\end{abstract}

Received date: September 10, 2017

Accepted date: October 20, 2017

Published date: October 24, 2017

Citation: D'Elia, C., et al. Functional Outcomes and Health Related Quality of Life after Artificial Urinary Sphincter Implantation: Evaluation with Validated Questionnaires. (2017) J Anesth Surg 4(2): 115- 120.

DOI: $10.15436 / 2377-1364.17 .092$

Keywords: Quality of life; Incontinence; Urinary sphincter

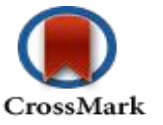

Abbreviations: AMS: American Medical System; AUS: Artificial Urinary Sphincter; HRQoL: health related quality of life; ICIQSF: International Consultation on Incontinence Questionnaire - short form; IQR: interquartile range; PGI-I: Patient Global Impression of Improvement; PPD: pad per day used; QoL: Quality of Life; RARP: Robot Assisted Radical Prostatectomy; RRP: Radical Retropubic Prostatectomy; TURP: Transurethral Prostate Resection; SD: standard deviation; SUI: stress urinary incontinence; VAS: visual analogue scale

\section{Introduction}

Several devices designed to control urinary incontinence in humans began to appear in the second half of the eighteenth century. Urinary incontinence is a, unfortunately not rare, sequela of several urologic procedures. This complication ranges from 4 
to $31 \%$ in Robot Assisted Radical Prostatectomy (RARP) ${ }^{[1]}$ and from 7 to $40 \%$ in Radical Retropubic Prostatectomy (RRP) ${ }^{[2]}$. With regard to Transurethral Prostate Resection (TUR-P), the gold standard treatment for benign prostatic hyperplasia, the most frequent benign neoplasm in $\operatorname{men}^{[3]}$ post operative late incontinence is absolutely rare $(<0.5 \%)^{[4]}$.

At the moment, the Artificial Urinary Sphincter (AUS), introduced in 1973 by the American Medical System Minneton$\mathrm{ka}, \mathrm{MN}, \mathrm{USA}^{[5]}$, is considered the gold standard treatment for stress urinary incontinence after prostate surgery; a Cochrane review conducted in 2014 documented that patients treated with AUS, iun comparison with those treated with injetable devices, are more likely to be continence, especially those with sever incontinence (OR 8.89) ${ }^{[6]}$.

This device provides good continence rate, ranging from 61 to $95 \%$, with a complication rate of about $12 \%$, as reported in a review of Hussain et al ${ }^{[7]}$. Moreover, AUS implantation can ameliorate quality of life of the patients undergoing the procedure, as reported by Trigo et al. The Colleagues used a VAS (visual analogue scale) score, that post operatively decreased from 5.0 to $1.4(\mathrm{P}<0.001)^{[8]}$. The purpose of our study was to assess efficacy and safety and quality of life outcomes in a series of patients treated with AUS AMS 800 (American Medical Systems, Minnetonka, Minn) at our urologic clinic.

\section{Materials and methods}

We prospectively collected and retrospectively reviewed the data of 37 patients who underwent placement of AUS type AMS 800 at our clinic. Indication for surgery was the stress urinary incontinence (SUI) after prostatic surgery, such as radical retropubic prostatectomy (RRP) or robot assisted robotic prostatectomy (RARP), transcapsular prostatic adenomectomy (Millin) or transurethral resection of the prostate (TURP). Inclusion criteria were as follows:

- age $>18$ years

- prostatic surgery performed $>6$ months before AMS 800 placement

- urodynamic confirmed severe SUI

- failure of conservative treatments (pelvic floor muscle training - biofeedback)

Exclusion criteria for the procedure were documented infravesical obstruction, detrus or over activity or under activity (urodynamic documented). Previous external beam radiotherapy or brachytherapy was not an exclusion criteria, unless performed in the previous 6 months. Pre and post operative SUI was evaluated using the daily pad use (PPD) and the Italian validated International Consultation on Incontinence Questionnaire - short form (ICIQ-SF) ${ }^{[9]}$, whereas patients comorbidity was assessed using the Charlson Comorbidity index ${ }^{[10]}$.

Health related quality of life and subjective satisfaction of the patients was evaluated with the Italian validated Patient Global Impression of Improvement (PGI-I) questionnaire ${ }^{[11,12]}$ ("Appendix"). Moreover, to assess the degree of personal satisfaction, patients were asked to rate on a scale from 0 to 100 their improvement and satisfaction after surgery and if they would recommend the procedure to a friend.

All patients underwent AMS 800 placement following a standard surgical procedure; all the procedures were performed by the same operator, skilled for the procedure, (thereby avoiding the risk of learning curves during the study ${ }^{[13]}$. Data obtained from the retrospective review included age, type of surgery, follow up (months), PPD (pads per day) used, PGI score. All these data were retrospectively analyzed and a comprehensive database was created.

\section{Statistical analysis}

Continuous variables with normal distribution were reported as mean \pm standard deviation (SD), whereas continuous variables with non-normal distribution were presented as medians and inter quartile ranges (IQR). The Mann-Whitney U test was used for comparison of continuous variables not for independent groups while the Wilcoxon Matched pair test was used for the analysis of pre- and post-operative variables. Moreover, Pearson's coefficient were used to evaluate the relationship between ICIQS-SF and PGI-I questionnaire and number and type of pads used.

Two-tailed $\mathrm{p}$ values $<0.05$ were considered statistically significant. All statistical analyses were performed with the software Statistical Package for Social Sciences software, version 20.0 (SPSS).

\section{Results}

We prospectively collected and retrospectively evaluated the data regarding 37 consecutive patients undergoing AMS 800 artificial sphincter placement. Table 1 summarizes the clinical characteristics of 37 patients who underwent placement intervention of USA type AMS 800 at our clinic from 2001 to 2015.

Table 1: Characteristics of the patients.

\begin{tabular}{|l|c|}
\hline Variable & N (\%) \\
\hline Age (mean \pm DS) in years & $68.8 \pm 5.3$ \\
\hline Previous procedure & $29(78.4 \%)$ \\
\hline RRP & $2(5.4 \%)$ \\
\hline RARP & $4(10.8 \%)$ \\
\hline TURP & $2(5.4 \%)$ \\
\hline Millin & $4(1-15)$ \\
\hline $\begin{array}{l}\text { Follow-up (median; range) in } \\
\text { years }\end{array}$ & \\
\hline $\begin{array}{l}\text { Pts dead at follow-up } \\
\text { Causes of death: }\end{array}$ & 4 \\
\hline - progression of prostate cancer & 2 \\
\hline - other cancer (lung) & 1 \\
\hline - other causes & 1 \\
\hline Revision surgery & 2 \\
\hline - Removal & 1 \\
\hline - Substitution & 1 \\
\hline
\end{tabular}

Mean age of the patient at time of AMS 800 placement was $68.8 \pm 5.3$ years. Majority of patients (29/37) underwent RRP, whereas only 2 underwent RARP and 8 (21.6\%) were treated with adjuvant radiotherapy (in 2 cases associated with chemotherapy). Eight of the 37 selected patients $(21.6 \%)$ had been subjected to post-operative radiotherapy (associated with chemotherapy in 2 cases).

During follow-up, removal of AMS 800 was required 
in a patient undergoing RRP, due to a "rejection" foreign body. In another patient, treated with RRP, the Artificial Urinary Sphincter AMS 800 has been replaced 2 years from his position because of the interruption of the connecting tubes (cut accidentally during an inguinal hernia repair surgery). Median preoperative PPD used was 4 (IQR 3-5), all of medium or large dimensions diapers, ranging from 3 to 7 PPD. $3 / 37$ patients, moreover, due to the continuous leakage, prefer to use an urocondom (Table 2).

After a median follow up of 4 years (range: 1 - $15 \mathrm{yrs}$ ), 4 patients were dead: 2 for progression of prostate cancer, 1 for other cancer (lung) and 1 for other causes. At the last follow up, median PPD used was 1, ranging from a minimum of 0 to a maximum of 3 PPD. Only a patient, because continuous urinary incontinence, wore an urocondom (Table 2).

Table 2: Comparison between PPD used before and after surgery.

\begin{tabular}{|l|c|c|}
\hline & PPD (median; IQR) & P \\
\hline Before surgery & $4(3-5)$ & 0.001 \\
\hline After surgery & $1(1-2)$ & \\
\hline
\end{tabular}

All the 33 patients alive at follow-up all agreed to undergo telephone interview with administration of the two questionnaires: the International Consultation on Incontinence Questionnaire-Short Form (ICIQ-SF) for the evaluation of the urinary continence and the Patient Global Impression of Improvement (PGI-I) for the evaluation of the Quality of Life (QoL) and the degree of personal satisfaction ${ }^{[9,11-12]}$, both validated in Italian.

To assess the degree of personal satisfaction, patients were asked to rate on a scale from 0 to 100 their improvement and satisfaction after surgery. Moreover, patients were asked if they would recommend the procedure to a friend. Table 3 shows the results regarding type of protective aids used for urinary incontinence after AMS 800 placement. Of the 33 patients interviewed, 24 patients were using small diapers, in most cases as "safety pad", while the remaining 8 were wearing medium-sized diapers and one an urocondom.

Table 3: Type and number of aids used by patients after surgery.

\begin{tabular}{|l|c|}
\hline Variable & N (\%) \\
\hline Diapers size: & $24(72.7 \%)$ \\
\hline Small & $8(21.6 \%)$ \\
\hline Medium-large & $1(2.7 \%)$ \\
\hline Urocondom & $1(1-2)$ \\
\hline Median PPD (IQR) & $20(60.6 \%)$ \\
\hline PPD & $13(39.4 \%)$ \\
\hline $\mathbf{0 - 1}$ &
\end{tabular}

A total of 20 patients employing 0 - 1 nappies a day and the remaining $\geq 2$ diaper a day (including in that group also the patient with urocondom). With regard to ICIQ-SF questionnaire, 4 patients $(12.5 \%)$ responded that they never lose urine and 22 $(68.76 \%)$ only during exercise and / or sneezing. Five patients $(15.6 \%)$ are losing always, whereas in 1 patient $(3.12 \%)$ IUS was associated with urgency.

The median score (IQR) of the ICIQ-SF questionnaire was equal to two $(2-7)$, with a normal value $<11$. The median score (IQR) of the PGI-1 questionnaire was equal to $1(1-2)$, documenting a good satisfaction of our patient after AMS 800 position. With regard to ICIQ-SF, the correlation between questionnaire score and type of diapers used was 0.77 , while that between ICIQ-SF score number of diapers used was 0.70 (Table 4).

Table 4: Correlation coefficient (CC) questionnaire scores and type and PPD, and number and type of aids worn continence.

\begin{tabular}{|l|l|c|}
\hline Questionnaire & Variable & Correlation Coefficient \\
\hline ICIQ-SF & Type of Pads & 0.77 \\
\hline ICIQ-SF & PPD & 0.7 \\
\hline PGI-I & Type of pads & 0.74 \\
\hline PGI-I & PPD & 0.63 \\
\hline
\end{tabular}

With regard to PGI-I, the correlation between questionnaire score and type of diapers was 0.74 , while that between PGI-I score and number of diapers used was of 0.63 , (Table 4) Comparing the median (IQR) of the scores related to both ICIQSF questionnaires and PGI-I with type (small or medium-large) and the number $(0-1$ or $\geq 2)$ of pads per day used, a best score in both questionnaires was associated with a smaller number of diapers and with the use of smaller diapers (Table 5).

Table 5: Association between questionnaire scores and type and PPD used.

\begin{tabular}{|l|l|l|l|}
\hline Variable & N & Median score (IQR) & P \\
\hline Type of pads & & ICI-Q & \\
\hline Small & 24 & $2(2-2)$ & 0.001 \\
\hline Medium-Large & 8 & $12(7.7-19)$ & \\
\hline Number of pads & & ICI-Q & \\
\hline 0 - 1 & 20 & $2(2-2)$ & 0.001 \\
\hline$>\mathbf{2}$ & 12 & $12(7.7-15.2)$ & \\
\hline Type of pads & & PGI-I & \\
\hline Small & 24 & $1(1-1)$ & 0.001 \\
\hline Medium-Large & 8 & $2.5(2-4)$ & \\
\hline Number of pads & & PGI-I & \\
\hline 0 - 1 & 20 & $1(1-1)$ & 0.001 \\
\hline$>\mathbf{2}$ & 12 & $2(1.7-3.2)$ & \\
\hline
\end{tabular}

As reported in Table 5, 20 patients used $0-1$ diapers / day, with a median score of ICIQ-SF of 2, while 12 patients used diapers $\geq 2$ / day with median score of 7.5. The 24 patients using small pads showed a median PGI-I score of 1, significatively higher in comparison with the score (2.5) of the patients using medium-large pads $(<0.5)$; similar results were obtained with regard to ICIQ-SF score (ICIQ-S median score 2 vs 12; $<<$ $0.05)$.

Table 6: Post operative subjective improvement and degree of satisfaction.

\begin{tabular}{|l|l|}
\hline & Median (IQR) \\
\hline 0 to 100 post operative improvement & $90(85-99)$ \\
\hline 0 to 100 post operative satisfaction & $99(90-100)$ \\
\hline
\end{tabular}


The 20 patients using 0 - 1 diaper per day showed median PGI-I score of 1, significatively lower in comparison with those patients using $<2$ diaper per day (PGI-I score $2 ; \mathrm{p}<0.5$ ); similar results are showed for ICIQ - SF score (2 VS 7.5; $\mathrm{p}<$ $0.5)$. All patients accepted to answer to the questions. With regard to the answer regarding the 0 to 100 improvement after surgery, median score was 90 , ranging from 20 to 100 (IQR 85-99), while concerning the satisfaction answer, median score was 99, ranging from 20 to 100 (IQR 90-100).

When we asked, "would you recommend the post to a friend? “, only 1 patient replied no, whereas all other patients responded yes.

\section{Discussion}

Our monocentric study documented that, at a median follow-up of 48 months patients who underwent AMS 800 placement present good results in terms of urinary continence, quality of life and degree of satisfaction. To our knowledge, our study is one of the few available in the literature, that used validated questionnaires like ICIQ-SF for the evaluation of urinary continence and the PGI-I for quality of life assessment; moreover, subjective satisfaction was evaluated with a simple question to the patients.

Consequently, our data are not directly comparable with those of other published series. The evidence in favor of the Artificial Urinary Sphincter type AMS 800 in the literature is based on several not high qualities, heterogeneous and not comparable, studies. Nevertheless, most of the literature studies did not used standard parameters for continence evaluation and definition; urinary continence was assessed evaluating number or type of pad used per day, or considering a nonstandard definition of "social continence ".

The questionnaires used are heterogeneous, like VAS score, ICIQ SF questionnaire, or patients were evaluated with not validated answer. In 2007 Lai et coll. retrospectively reviewed a large series of 218 patients undergoing artificial urinary sphincter implantation (narrow backed cuff) at a single institution with a mean follow up of 36.5 months, but patients were not evaluated with validated questionnaires regarding continence or quality of life $(\mathrm{QoL})^{[14]}$, as well in another multicenter prospective survey of Mottet et al, conducted on 103 male patients with bulbar cuff, with a reported success rate of about $61 \%{ }^{[15]}$.

Moreover, Ramsay et al and Singh et al, in two retrospective papers, not using validate questionnaires, reported high success rates of $100 \%$ an $96 \%(27 / 27$ pts and $20 / 21$, respectively, achieving "social continence") and 20/21 patients dry and improved ${ }^{[16,17]}$, with significatively higher success rats in comparison with our series and slightly lower follow up. On the other hand, Trigo Rocha et al prospectively evaluated 40 patients using PPD and VAS score $(0-6)$ with the aim to analyze the impact of the incontinence on QOL of the patients; $50 \%$ of the patients was completely dry, with PPD count dropping from $4.0 \pm 0.9$ to $0.6 \pm 1.1$ pads per day and impact of incontinence in QoL measured by the VAS dropping from $5.0 \pm 0.7$ to $1.4 \pm$ 0.9 (P 0.001$)^{[18]}$, according to the result of our little series, with a PPD dropping from $4(3-5)$ to $1(0-3)$.

Moreover, O Connor et coll evaluated a group of 47 men ( 25 single cuff) undergoing AUS placement using the Incontinence Impact Questionnaire Short Form (IIQ-7), postop- erative PPD, chart review and patient/family interview, documenting a success rate of $61 \%(0-1$ PPD) in the double cuff cohort and a mean IIQ-7 ranging from 16.3 to $6.4^{[19]}$. Nevertheless, Imamoglu et al, in a randomized controlled trial comparing macroplastique and AUS, evaluating patients with a QoL scale, documented a dry rate of $82 \%$ with a PPD dropping from 2.27 to 0.36 and a QoL scale from 33.3 to 9.2 in the "severe" incontinence group ${ }^{[20]}$.

According to the several definition of continence, success rate varies in literature from $4.1 \%$ of "dry" patients reported in the retrospective series of $\mathrm{O}^{\prime}$ Connor et $\mathrm{al}^{[19]}$, using a validated questionnaire, to $100 \%$ improved patient of Ramsay et $\mathrm{al}^{[16]}$, not using a validated questionnaire. In our retrospective series, the majority of patients in our study used 0-1 PPD after the procedure and most of these pads were of small size, used as a safety pad. Moreover, $12.5 \%$ of our patients are completely dry (defined as patients who report never lose urine, regardless of whether or not they should declare to use one pad per day as a safety device).

With regard to patients who reported losing urine, in most cases $(68.76 \%)$ this happened during physical activity, or during coughing or sneezing. This data is also known in the literature and explained with the fact that a sudden increase in abdominal pressure also would lead to a sudden increase in pressure around the cuff, causing it to lose effectiveness at that time. Most of the patients in our study stated that their QoL was extremely or much improved after the surgery, as documented by median score of PGI-I equal to $1(1-2)$ and by the answers regarding procedure satisfaction or recommendation to a friend.

The main limitation of our study is the cross-sectional design relating to a retrospective cohort of patients, but the lack of pre operative QoL assessment of quality of life before surgery prevents intervention impact analysis on the same. Our monocentric series is, moreover, not particularly large, but has the advantage, that all surgeries were performed by a single operator, expert in this type of surgery. The main advantage of our study is, on the other hand, that patients were evaluated with validated questionnaires, only rarely used in the studies available in the literature.

AS highlighted by Van der As er al in their review, in the future they would serve prospective larger and collaborative studies ${ }^{[21]}$, using validated continence, QoL and sexual function questionnaires, in order to assess both patient's baseline both follow uppost operative functional results.

\section{Conclusions}

In our experience, at a median follow-up of 48 months, the AMS 800 AUS ensures good results in terms of urinary continence, with a significant reduction in the number of PPD, and a satisfactory quality of life. The majority of patients continue to wear a small pad to purely precautionary purposes, since the diaper is often dry to the exchange. The chances of urine leakage occur in conjunction with physical activity, coughing or sneezing. Our patients are happy and satisfied with the intervention and would recommend to their friends. 


\section{Appendix}

\begin{tabular}{|l|}
\hline \multicolumn{1}{|c|}{ ICIQ-SF Questionnaire } \\
\hline Ha Perdite di urina? SI No \\
\hline SOMMA PUNTEGGI $1+2+3$ \\
\hline 1) Con quale frequenza le capita di perdere urina? \\
\hline -Mai (0) \\
\hline -Talvolta (1) \\
\hline -Regolarmente (2) \\
\hline -Sempre (3) \\
\hline
\end{tabular}

2) Secondo la sua personale opinione quanta urina perde comunemente?

\begin{tabular}{|l|}
\hline 0- Non perdo urina \\
\hline 2- Perdo piccole quantità di urina \\
\hline 4- Perdo modeste quantità di urina \\
\hline 6- Perdo abbondanti quantità di urina \\
\hline
\end{tabular}

3) nel complesso le sue perdite quanto influiscono negativamente nella sua vita

$(0=$ per niente, $10=$ moltissimo $)$

$$
1-(0-1)
$$

$3-(2-3)$

5- (4-5)

7- (6-7)

9- (8-9)

10- (10)

In quali circostanze perde urina?

-prima di arrivare in bagno

-quando tossisco o starnutisco

-durante il sonno

-durante l'attività fisica

-una volta rivoestito/a dopo aver urinato

-senza ragioni particolari

-sempre

\section{PGI-I}

PATIENT GLOBAL IMPRESSION OF IMPROVEMENT

Barrare la casella che descrive in maniera più adeguata come si sente dopo essere stato sottoposto alla terapia/intervento

1. estremamente migliorato

2. molto migliorato

3. lievemente migliorato

4. nessun cambiamento

5. lievemente peggiorata

6. molto peggiorata

7. estremamente peggiorata

(*)Il suddetto questionario deve essre compilato solo in occasione della visita di controllo

\section{UTILIZZO DI AUSILI}

Occorre descrivere il numero di ausili utilizzati al giorno e la tipologia

$\mathrm{P}=$ piccoli (salvaslip)

$\mathrm{M}=$ medi (pannolini piccoli o da flusso leggero)

$\mathrm{G}=$ grandi (pannolini medio-grandi da flusso abbondante)

"Back to Top." 


\section{References}

1. Ficarra, V., Novara, G., Rosen, R.C., et al. A Systematic review and meta-analysis of studies reporting urinary continence recovery after robot-assisted radical prostatectomy. (2012) Eur Urol 62(3): 405-417.

Pubmed | Crossref | Others

2. Ficarra, V., Novara, G., Artibani, W., et al. Retropubic, laparoscopic, and robot-assisted radical prostatectomy: a systematic review and cumulative analysis of comparative studies. (2009) Eur Urol 55(5): $1037-$ 1063.

Pubmed | Crossref | Others

3. AUA Practice Guidelines Committee: AUA guideline on management of benign prostatic hyperplasia (2003). Chapter 1: Diagnosis and treatment recommendations. (2003) J Urol 170: 530-547.

Pubmed | Crossref | Others

4. Rassweiler, J., Teber, D., Kuntz, R., et al. Complications of transurethral resection of the prostate (TURP)- incidence, management, and prevention. (2006) Eur Urol 50(5): 969-979.

Pubmed | Crossref | Others

5. Scott, F.B., Bradley, W.E., et al. "Treatment of urinary incontinence by implantable prosthetic sphincter,". (1973) Urology 1(3): 252-259.

Pubmed | Crossref | Others

6. Silva, L.A., Andriolo, R.B., Atallah, Á.N., et al. Surgery for stress urinary incontinence due to presumed sphincter deficiency after prostate surgery. (2014) Cochrane Database Syst Rev 27(9): CD008306.

Pubmed | Crossref | Others

7. Hussain, M., Greenwell, T.J., Venn, S.N., et al. The current role of the artificial urinary sphincter for the treatment of urinary incontinence. (2005) J Urol 174(2): 418-424.

Pubmed | Crossref | Others

8. Trigo Rocha, F., Gomes, C.M., Mitre, A.I., et al. A prospective study evaluating the efficacy of the artificial sphincter AMS 800 for the treatment of postradical prostatectomy urinary incontinence and the correlation between preoperative urodynamic and surgical outcomes. (2008) Urology 71(1): 85-89.

Pubmed | Crossref | Others

9. Espuna-Pons, M., Dilla, T., Castro, D. Analysis of the value of the ICIQ-UI SF questionnaire and stress test in the differential diagnosis of the type of urinary incontinence. (2007) Neurourol Urodyn 26: 836841.

Pubmed | Crossref | Others

10. Charlson, M., Szatrowski, T.P., Peterson, J., et al. Validation of a combined comorbidity index. (1994) J Clin Epidemiol 47: 1245-1251. Pubmed | Crossref | Others
11. Yalcin, I., Bump, R.. Validation of two Global Impression Questionnaires for incontinence. (2003) Am J Obstet Gyn 189: 98-101.

Pubmed | Crossref | Others

12. Tincello, D.G., Owen, R.K., Slack, M.C., et al. Validation of the patient global impression scales for use in detrusor overactivity: secondary analysis of the RELAX Study. (2013) BJOG 120(2): 212-216. Pubmed $\mid$ Crossref $\mid$ Others

13. Lai, H.H. Incontinence: what is the learning curve for artificial urinary sphincter surgery? (2011) Nat Rev Urol 8(9): 475-476.

Pubmed | Crossref | Others

14. Lai, H.H., Hsu, E.I., Teh, B.S., et al. 13 years of experience with artificial urinary sphincter implantation at Baylor College of Medicine. (2007) J Urol 177: 1021-1025.

Pubmed | Crossref | Others

15. Mottet, N., Boyer, C., Chartier-Kastler, E., et al. Artificial urinary sphincter AMS 800 for urinary incontinence after radical prostatectomy: the French experience. (1998) Urol Int (Suppl 2): 25-29.

Pubmed $\mid$ Crossref $\mid$ Others

16. Ramsay, A.K., Granitsiotis, P., Con, I.G. The use of artificial urinary sphincter in the west Scotland: a single center 10-year experience. (2007) Scott Med J 52(2): 14-17.

Pubmed | Crossref | Others

17. Singh, G., Thomas, D.G. Artificial urinary sphincter for post prostatectomy incontinence. (1996) Br J Urol 77: 248-251.

Pubmed | Crossref | Others

18. Gomes, C.M., Broderick, G.A., Sanchez-Ortiz, R.F., et al. Artificial urinary sphincter for post-prostatectomy incon- tinence: impact of prior collagen injection on cost and clinical outcome. (2000) J Urol 163: 87-90.

Pubmed | Crossref | Others

19. O'Connor, R.C., Lyon, M.B., Guralnick, M.L., et al. Long-term follow- up of single versus double cuff artificial urinary sphincter insertion for the treatment of severe postprostatectomy stress urinary incontinence. (2008) Urology 71(1): 90-93.

Pubmed | Crossref | Others

20. Imamoglu, Ma., Tuygun, C., Bakirtas, H., et al. The comparison of artificial urinary implantation and endouretral Macroplastique injection for the tratment of postprostatectomy incontinence. (2005) Eur Urol 47(2): 209-213.

Pubmed | Crossref | Others

21. Van der, A.F., Drake, M.J, Kasyan G.R, et al. The Artificial Urinary Sphincter After a Quarter of Century: A Critical Systematic Review of its Use in Male Non-neurogenic Incontinence. (2013) Eur Urol 63: 681689.

Pubmed | Crossref | Others
Ommega Online Publishers

Journal Title: Journal of Anesthesia and Surgery (JAS)

Journal Short Name: J Anesth Surg
Journal ISSN: 2377-1364

E-mail: anestheisa@ommegaonline.com

Website: www.ommegaonline.org 